Software Engineering

$\int$ SEN $\begin{aligned} & \text { Software ENgineering } \\ & \text { Coalgebraic modal logic of finite rank }\end{aligned}$
A. Kurz, D. Pattinson

Report SEN-R0222 November 30, 2002 
CWI is the National Research Institute for Mathematics and Computer Science. It is sponsored by the Netherlands Organization for Scientific Research (NWO).

$\mathrm{CWI}$ is a founding member of ERCIM, the European Research Consortium for Informatics and Mathematics.

CWI's research has a theme-oriented structure and is grouped into four clusters. Listed below are the names of the clusters and in parentheses their acronyms.

Probability, Networks and Algorithms (PNA)

\section{Software Engineering (SEN)}

Modelling, Analysis and Simulation (MAS)

Information Systems (INS)

Copyright @ 2001, Stichting Centrum voor Wiskunde en Informatica

P.O. Box 94079, 1090 GB Amsterdam (NL)

Kruislaan 413, 1098 SJ Amsterdam (NL)

Telephone +31205929333

Telefax +31 205924199 


\title{
Coalgebraic Modal Logic of Finite Rank
}

\author{
Alexander Kurz \\ email: kurz@cwi.nl \\ CWI, Amsterdam, The Netherlands \\ Dirk Pattinson \\ email: pattinso@informatik.uni-muenchen.de \\ Institut für Informatik, Ludwig-Maximilians-Universität München, Germany
}

\begin{abstract}
This paper studies coalgebras from the perspective of finite observations. We introduce the notion of finite step equivalence and a corresponding category with finite step equivalence-preserving morphisms. This category always has a final object, which generalises the canonical model construction from Kripke models to coalgebras. We then turn to logics whose formulae are invariant under finite step equivalence, which we call logics of rank $\omega$. For these logics, we use topological methods and give a characterisation of compact logics and definable classes of models.
\end{abstract}

2000 Mathematics Subject Classification: 03B45

1998 ACM Computing Classification System: F.3.1, D.2.4

Keywords and Phrases: Modal logic, coalgebra, compactness, definability,

Note: An earlier version of this paper has been published as [13]. 


\section{Introduction}

Coalgebras for an endofunctor $T$ on Set encompass many types of state base systems, including Kripke models and frames, labelled transition systems, Mooreand Mealy automata and deterministic systems, see e.g. Rutten [28]. The research on modal logics as specification languages for coalgebras began with Moss [20] and was taken up in e.g. [17, 27, 25, 8,9].

The relationship between modal logic and coalgebras has been explained in [15] as follows. If $Z$ denotes the carrier of the final coalgebra, we can consider the semantics of a modal formula $\varphi$ as the subset $\llbracket \varphi \rrbracket \subseteq Z$ of states which satisfy $\varphi$. Intuitively, the elements of $Z$ are behaviours, and every modal formula $\varphi$ determines a set of behaviours which satisfy $\varphi$. In case the logic is fully expressive in the sense that it allows to define all subsets of $Z$, we can identify modal formulae with subsets of $Z$, resulting in an algebraic approach to investigate modal logics, see $[15,16]$.

In general however, finitary modal logics are not fully expressive. It is the main issue of this paper to present a semantic representation, which fits finitary logics as nicely as the representation as subsets of the final model suits fully expressive logics.

We use the so-called terminal sequence $\left(T^{n} 1\right)$ of the underlying endofunctor to capture the notion of finitely observable behaviour. The terminal sequence can be understood as approximating the final coalgebra, see [2]. Intuitively, the elements of the $n$-th approximant represent the behaviour that can be observed in $n$ transition steps. Following [21,22], we represent the semantics of a modal formula $\varphi$ of rank $n$ as subset $\llbracket \varphi \rrbracket_{n} \subseteq T^{n} 1$.

The terminal sequence also gives rise to a notion of finite step equivalence. Intuitively, two processes are $n$-step equivalent iff they show the same $n$-step behaviour, that is, if their projections into $T^{n} 1$ coincide. The main novelty of the paper is probably the introduction of the category $\operatorname{Beh}_{\omega}(T)$ that has coalgebras as objects and functions that preserve finite-step behaviours as morphisms (Section 3). This paper argues that the role of $\operatorname{Beh}_{\omega}(T)$ for finitary logics is the same as that of Coalg $(T)$ for fully expressive logics. In Section 4, we show that $\operatorname{Beh}_{\omega}(T)$ always has a final object, the subsets of which represent formulas of finitary logics. Moreover, we show that the final object in $\operatorname{Beh}_{\omega}(T)$ generalises the canonical model construction from Kripke models to coalgebras.

In Section 4, we begin the study of logics whose formulae are invariant under finite step equivalence. These logics are called logics of rank $\omega$. In case that the semantics of every formula can be represented as subset $\llbracket \varphi \rrbracket_{n} \subseteq T^{n} 1, n<\omega$, we speak of logics of finite rank. Whereas fully expressive modal logics allow to express all predicates of the carrier of the final coalgebra, logics of rank $\omega$ do not allow to express all predicates on the final object of $\operatorname{Beh}_{\omega}(T)$. This is the reason to consider topologies on coalgebras. The main idea here is that clopen subsets are precisely the predicates which can be expressed through a single formula.

This topology is then used to prove compactness and definability results. Section 7 shows that - under a mild hypothesis satisfied by all examples of interest - a logic of finite rank is compact iff the functor $T$ weakly preserves 
the limit of the finite part $\left(T^{n} 1\right)_{n<\omega}$ of the terminal sequence. Section 8 characterises classes of coalgebras, which are definable by a logic of rank $\omega$, as being closed under images, subcoalgebras, coproducts and topological closure.

\section{Preliminaries and Notation}

Throughout the paper, $T$ denotes an endofunctor on the category of sets and functions. A $T$-coalgebra is a pair $(C, \gamma)$ where $C$ is a set and $\gamma: C \rightarrow T C$ is a function. A coalgebra morphism $f:(C, \gamma) \rightarrow(D, \delta)$ is a function $f: C \rightarrow D$ such that $\delta \circ f=T f \circ \gamma$. The category of $T$-coalgebras and coalgebra morphisms is denoted by Coalg $(T)$. Given two $T$-Coalgebras $(C, \gamma)$ and $(D, \delta)$, two states $c \in C$ and $d \in D$ are called behaviourally equivalent, if they can be identified by a morphism of coalgebras, i.e. if there exists $(E, \epsilon) \in \operatorname{Coalg}(T), f:(C, \gamma) \rightarrow(E, \epsilon)$ and $g:(D, \delta) \rightarrow(E, \epsilon)$ with $f(c)=g(d)$. If Coalg $(T)$ has a final object $(Z, \zeta)$ and $!_{C}:(C, \gamma) \rightarrow(Z, \zeta)$ and $!_{D}:(D, \delta) \rightarrow(Z, \zeta)$ denote the unique morphisms into the final object, this is clearly equivalent to $!_{C}(c)=!_{D}(d)$. We think of a coalgebra $(C, \gamma)$ together with a state $c$ as a process and call $!_{C}(c)$ its behaviour.

Example 1.1. (Streams) For a set $D$ consider $T X=D \times X$. Given a coalgebra $\gamma=\langle$ head, tail $\rangle: C \rightarrow D \times C$ the behaviour of an element $c \in C$ is the infinite list (head $(c)$, head $(\operatorname{tail}(c))$,

head $(\operatorname{tail}(\operatorname{tail}(c))), \ldots)$. Accordingly, the final coalgebra $\left(D^{\omega},\langle\right.$ head, tail $\left.\rangle\right)$ is given by the infinite lists over $D$.

Example 1.2. (Kripke models) Suppose Prop is a countably infinite set and $T X=\mathcal{P} X \times \mathcal{P}$ Prop. Then $T$-coalgebras are in 1-1 correspondence with Kripke models and behavioural equivalence coincides with bisimilarity.

We have seen that the final coalgebra (if it exists) defines a notion of behaviour. In general, every state of the final coalgebra represents an infinite amount of information. This paper suggests a framework to study finitely observable properties of systems. Hence the final coalgebra (containing the infinite behaviours of all coalgebras) has to be replaced by finitary approximations. These approximations are provided by the (finitary part) of the so-called terminal sequence of the underlying endofunctor $T$.

\subsection{The Terminal Sequence}

The terminal sequence can be thought of as approximating the final coalgebra. The following definition has been taken from [31].

The terminal sequence of $T$ is an ordinal indexed sequence of sets $\left(Z_{n}\right)$ together with a family $\left(p_{m}^{n}\right)_{m \leq n}$ of functions $p_{m}^{n}: Z_{n} \rightarrow Z_{m}$ for all ordinals $m \leq n$ such that

- $Z_{n+1}=T Z_{n}$ and $p_{m+1}^{n+1}=T p_{m}^{n}$ for all $m \leq n$

- $p_{n}^{n}=i d_{Z_{n}}$ and $p_{k}^{n}=p_{k}^{m} \circ p_{m}^{n}$ for $k \leq m \leq n$. 
- The cone $\left(Z_{n},\left(p_{m}^{n}\right)\right)_{m<n}$ is limiting whenever $n$ is a limit ordinal.

Thinking of $Z_{n}$ as the $n$-fold application of $T$ to the limit $1=\{0\}$ of the empty diagram, we write $Z_{n}=T^{n} 1$ in the sequel. Intuitively, $T^{n} 1$ represents behaviour which can be observed in $n$ steps. If, for example $T X=D \times X$, then $T^{n} 1 \cong D^{n}$ contains all lists of length $n$.

Note that every coalgebra $(C, \gamma)$ gives rise to a cone $\left(C,\left(\gamma_{n}: C \rightarrow T^{n} 1\right)\right)$ over the terminal sequence:

Definition 1.3. If $(C, \gamma) \in \operatorname{Coalg}(T)$, define $\gamma_{n}: C \rightarrow T^{n} 1$ to be $T \gamma_{m} \circ \gamma$, if $n=m+1$ is a successor ordinal, and the unique map satisfying $\gamma_{m}=p_{m}^{n} \circ \gamma_{n}$ for all $m<n$, if $n$ is a limit ordinal.

We will often use without further mentioning the following easy

Proposition 1.4. Let $n$ be an ordinal.

1. Let $f:(C, \gamma) \rightarrow(D, \delta)$ be a coalgebra morphism. Then $\delta_{n} \circ f=\gamma_{n}$.

2. Let $(C, \gamma) \in \operatorname{Coalg}(T)$. Then $p_{n}^{n+1} \circ T\left(\gamma_{n}\right) \circ \gamma=\gamma_{n}$.

\section{Introductory Examples}

For illustration and motivation of our later development, we discuss two different logics in detail. The main claim which we want to substantiate is that modal formulas can be semantically represented as subsets of $T^{n} 1$, where $n$ is the rank of the formula.

\subsection{Propositional Modal Logic}

This section argues that modal formulas of finite rank, interpreted over coalgebras, have a natural representation as subsets of some $T^{n} 1$, where $n \in \omega$ is a finite ordinal. We start by re-considering Example 1.2 and show, that a formula of rank $n$ can be represented as subset of $T^{n} 1$.

Suppose $T X=\mathcal{P} X \times \mathcal{P}$ Prop as in Example 1.2. Then $T$-coalgebras are Kripke models, which is why we use propositional modal logic to describe properties of $T$-coalgebras. We denote the language of propositional modal logic by $\mathcal{M L}$, that is, $\mathcal{M L}$ is the least set according to the grammar

$$
\mathcal{M L} \ni \varphi, \psi::=\mathrm{ff}|p| \varphi \rightarrow \psi \mid \square \varphi
$$

where $p \in$ Prop ranges over the set of atomic propositions.

Given a $T$-coalgebra $(C, \gamma)$, the semantics $\llbracket \varphi \rrbracket=\llbracket \varphi \rrbracket_{(C, \gamma)} \subseteq C$ of a modal formula $\varphi \in \mathcal{M L}$ is then inductively defined by 
- $\llbracket \mathbb{f} \rrbracket=\emptyset$

- $\llbracket p \rrbracket=\left\{c \in C \mid p \in \pi_{2} \circ \gamma(c)\right\}$
- $\llbracket \varphi \rightarrow \psi \rrbracket=(C \backslash \llbracket \varphi \rrbracket) \cup \llbracket \psi \rrbracket$

- $\square \varphi \rrbracket=\left\{c \in C \mid \pi_{1} \circ \gamma(c) \subseteq \llbracket \varphi \rrbracket\right\}$

The meticulous reader is invited to check that this definition coincides with the usual semantics of propositional modal logic (cf eg [7]). Given a formula $\varphi \in \mathcal{M L}$, the rank of $\varphi$, which represents the nesting depth of $\square$-operators, is then given inductively by $\operatorname{rank}(\mathrm{ff})=0, \operatorname{rank}(\varphi \rightarrow \psi)=\max \{\operatorname{rank}(\varphi), \operatorname{rank}(\psi)\}$, $\operatorname{rank}(p)=1$ for $p \in \operatorname{Prop}, \operatorname{rank}(\square \varphi)=\operatorname{rank}(\varphi)+1$.

Semantically, the rank can be thought of the number of transition steps, a formula contains information about. A similar intuition applies to the approximants $T^{n} 1$ of the endofunctor: we think of predicates on $T^{n} 1$ as representing behaviour which can be observed in $n$ transition steps. The following proposition makes this relationship precise:

Proposition 2.1. Suppose $\varphi \in \mathcal{M L}$ has rank n. Then there exists $t \subseteq T^{n} 1$ such that $\llbracket \varphi \rrbracket_{(C, \gamma)}=\gamma_{n}^{-1}(t)$ for all $(C, \gamma) \in \operatorname{Coalg}(T)$.

Proof. By induction on the structure of formulas: For $\varphi=\mathrm{ff}$ evidently $\llbracket \varphi \rrbracket=$ $\gamma_{0}^{-1}(\emptyset)$. For the case $\varphi=p$ for $p \in$ Prop let $t=\{(\{0\},\{p\})\} \subseteq T 1$ (we assume $1=\{0\})$. Then $\llbracket p \rrbracket=\gamma_{1}^{-1}(t)$. If $\varphi, \psi \in \mathcal{M L}$ with $\operatorname{rank}(\varphi)=n, \operatorname{rank}(\psi)=m$, put $k=\max \{n, m\}$ and assume that $\llbracket \varphi \rrbracket=\gamma_{n}^{-1}(t), \llbracket \psi \rrbracket=\gamma_{m}^{-1}(s)$. For $u=$ $\left(T^{k} 1 \backslash\left(p_{n}^{k}\right)^{-1}(t)\right) \cup\left(p_{m}^{k}\right)^{-1}(s) \subseteq T^{k} 1$, the fact that $\left(C,\left(\gamma_{n}\right)\right)$ is a cone over the terminal sequence implies that $\llbracket \varphi \rightarrow \psi \rrbracket=\gamma_{k}^{-1}(u)$.

For the most interesting case $\varphi=\square \psi$ consider the operation defined by

$$
\lambda(X)(\mathfrak{x})=\left\{\left(\mathfrak{x}^{\prime}, \mathfrak{a}\right) \in \mathcal{P}(X) \times \mathcal{P}(\text { Prop }) \mid \mathfrak{x}^{\prime} \subseteq \mathfrak{x}\right\}
$$

where $X$ is a set and $\mathfrak{x} \subseteq X$. An easy calculation shows, that we can rephrase the semantics of the $\square$-operator as $\llbracket \square \psi \rrbracket=\gamma^{-1} \circ \lambda(C)(\llbracket \psi \rrbracket)$. Now assume that $\psi$ has rank $n$ with $\llbracket \psi \rrbracket=\gamma_{n}^{-1}(s)$. Put $t=\lambda\left(T^{n} 1\right)(s)$. Then $\llbracket \square \psi \rrbracket=\gamma_{n+1}^{-1}(t)$ follows from the fact that $\lambda: 2 \rightarrow 2 \circ T$ is a natural transformation, where 2 denotes the contravariant powerset functor.

This shows, that formulas of rank $n$ correspond to subsets of $T^{n} 1$ of the terminal sequence of $T$. In the proof of the above proposition, we used the fact that atomic propositions can be represented as subsets of $T^{n} 1$ and that the semantics of the $\square$-operator can be formulated in terms of a natural transformation $2 \rightarrow 2 \circ T$. Such natural transformations are often called predicate liftings and have been used by a number of authors [27, 25, 8, 9] to describe the semantics of modal logics over coalgebras. We thus obtain a wealth of examples for arbitrary endofunctors if we consider modal logics, where atomic propositions can be represented as subsets of $T 1$ and modal operators are interpreted using predicate liftings, see [21].

\subsection{Linear Temporal Logic on Streams}

Linear temporal logic $\mathcal{L} \mathcal{T} \mathcal{L}$ (see eg. $[12,18]$ ) is a temporal logic to describe properties of infinite runs of programs, i.e. streams. We use $T$-coalgebras for 
$T X=X \times \mathcal{P}$ Prop, with Prop countably infinite, as semantics. This is a slight deviation from the standard semantics, which is given in terms of infinite sequences of subsets of Prop, see Example 1.1 for discussion. The language $\mathcal{L} \mathcal{T} \mathcal{L}$ of linear temporal logic is the least set according to the grammar

$$
\mathcal{L} \mathcal{T} \mathcal{L} \ni \varphi, \psi::=\mathrm{ff}|p| \varphi \rightarrow \psi|\bigcirc \varphi| \square \varphi
$$

where $p \in$ Prop ranges over the set of atomic propositions. We read $\bigcirc$ as 'next' and $\square$ as 'always'. Given a $T$-coalgebra $(C, \gamma)$, we define the semantics $\llbracket \varphi \rrbracket=\llbracket \varphi \rrbracket_{(C, \gamma)}$ of an $\mathcal{L} \mathcal{T} \mathcal{L}$-formula $\varphi$ inductively by

$$
\text { - } \ \varphi \rrbracket=\left\{c \in C \mid \pi_{1} \circ \gamma(c) \in \llbracket \varphi \rrbracket\right\} \quad \text { - } \square \varphi \rrbracket=\bigcap_{n<\omega} \llbracket \bigcirc^{n} \varphi \rrbracket
$$

where $\bigcirc^{n}$ stands for a sequence of $n \bigcirc$-operators and the semantics of boolean operators and atomic propositions is as in the previous example. In contrast to the previous example, not all formulas can be represented as subsets of some approximant $T^{n} 1$ :

Example 2.2. Let $p \in \operatorname{Prop}$ and $\varphi=\square p$. Then there is no $n<\omega$ and $t \subseteq T^{n} 1$ with $\llbracket \varphi \rrbracket_{(C, \gamma)}=\gamma_{n}^{-1}(t)$ for all $(C, \gamma) \in \operatorname{Coalg}(T)$.

We can, however, represent every formula as subset of $T^{\omega} 1 \cong(\mathcal{P} \text { Prop })^{\omega}$ :

Proposition 2.3. For all $\varphi \in \mathcal{L T L}$ there is $t \subseteq T^{\omega} 1$ such that $\llbracket \varphi \rrbracket_{(C, \gamma)}=$ $\gamma_{\omega}^{-1}(t)$.

Proof. Consider $(K, \kappa)=\left((\mathcal{P P r o p})^{\omega},\langle\right.$ head, tail $\left.\rangle\right)$. Then $K \cong T^{\omega} 1$ and $\llbracket \varphi \rrbracket_{(C, \gamma)}=$ $\gamma_{\omega}^{-1}\left(\llbracket \varphi \rrbracket_{(K, \kappa)}\right)$.

Note that, in the proof of the above proposition, $(K, \kappa)$ is actually the final $T$-coalgebra.

\section{$3 \quad$ Finite Step Equivalence and the Category $\operatorname{Beh}_{\omega}(T)$}

The previous section has shown, that, for logics interpreted via predicate liftings, formulas of finite rank can be represented as subsets of the elements $T^{n} 1$ of $T$ 's terminal sequence. For the remainder of the exposition, we take a semantical view and take subsets of the $T^{n} 1$ as representing formulas of finite rank; this allows us consider logics for coalgebras in broad generality, without making a commitment to any particular syntax.

We begin with introducing a notion of equivalence on states, which reflects the fact that two states cannot be distinguished by a predicate of finite rank.

Definition 3.1. Let $n$ be an ordinal and suppose $\mathrm{C}=(C, \gamma), \mathrm{D}=(D, \delta) \in$ $\operatorname{Coalg}(T)$. For $c \in C$ we call $\gamma_{n}(c)$ the $n$-step behaviour of $c$.

1. Two states $(c, d) \in C \times D$ are called $n$-step equivalent, denoted by $c \sim_{n} d$, if $\gamma_{n}(c)=\delta_{n}(d)$. We call $c$ and $d$ finite step equivalent if $c \sim_{n} d$ for all $n<\omega$. 
2. The systems $\mathrm{C}$ and $\mathrm{D}$ are $n$-step equivalent, denoted by $\mathrm{C} \sim_{n} \mathrm{D}$, if $\gamma_{n}(C)=$ $\delta_{n}(D)$. They are called finite step equivalent, denoted by $\mathrm{C} \sim<\omega$, if $\mathrm{C} \sim_{n}$ D for all $n<\omega$.

Under the assumption that the final coalgebra exists, two states of coalgebras are behaviourally equivalent, if they are identified by the unique morphism into the final coalgebra. As shown in [2], this is equivalent to $\gamma_{n}(x)=\gamma_{n}(y)$ for all ordinals $n$. Finite step equivalence, as introduced above, restricts the validity of this equation to finite ordinals. Note that $c, d$ are finite step equivalent iff $c \sim_{\omega} d$. In the context of modal logic, (that is, for $T X=\mathcal{P} X \times \mathcal{P}$ Prop), finite step equivalence is (a slight variation of) the bounded bisimulation of modal logic as studied in [6].

The next proposition clarifies on the relationship between finite step equivalence and behavioural equivalence on states of coalgebras:

Proposition 3.2. Suppose $(C, \gamma),(D, \delta) \in \operatorname{Coalg}(T)$ and $(c, d) \in C \times D$.

1. If $c$ and $d$ are behaviourally equivalent, then they are finite step equivalent.

2. If $T$ is $\omega$-accessible, then $c$ and $d$ are behaviourally equivalent if and only if they are finite step equivalent.

Proof. The first claim is an easy induction, the second claim follows by terminal sequence induction, see Worrell [31] or Theorem 3.4 of [23].

In order to obtain an example of two states, which are finite step equivalent, but not behaviourally equivalent, one therefore needs to consider a functor, which is not $\omega$-accessible.

Example 3.3. Let $T X=\mathcal{P}(X)$ and consider $C=\omega+2, \gamma(c)=\cup c$. One shows by induction that $c, d$ are $n$-step equivalent if $c \cap n=d \cap n$, hence $\omega$ and $\omega+1$ are finite step equivalent. If they were behaviourally equivalent, one would obtain $\gamma_{\omega+1}(\omega)=\gamma_{\omega+1}(\omega+1)$, which is not the case.

While finite step equivalence and $\omega$-step equivalence defines the same notion on states, note that $\omega$-step equivalence is in general not implied by finite step equivalence for systems:

Example 3.4. Let $T X=\{a, b\} \times X, C$ the final coalgebra with carrier $\{a, b\}^{\omega}$ and D the subcoalgebra with carrier $\left\{s \cdot a^{\omega}: s \in\{a, b\}^{*}\right\}$. Then $\mathrm{C}$ and $\mathrm{D}$ are finite step equivalent, but not $\omega$-step equivalent.

In the category Coalg $(T)$, morphisms can easily seen to preserve behavioural equivalence. We now introduce the category $\operatorname{Beh}_{\omega}(T)$, the morphisms of which are only required to preserve finite step equivalence. Recall that $\delta_{\omega} \circ f=\gamma_{\omega}$ iff $\delta_{n} \circ f=\gamma_{n}$ for all $n<\omega$ whenever $(C, \gamma),(D, \delta) \in \operatorname{Coalg}(T)$ and $f: C \rightarrow D$ is any function.

Definition 3.5 $\left(\operatorname{Beh}_{\omega}(T)\right)$. The category $\operatorname{Beh}_{\omega}(T)$ has $T$-coalgebras as objects. Morphisms $f:(C, \gamma) \rightarrow(D, \delta)$ of $\operatorname{Beh}_{\omega}(T)$ are functions $f: C \rightarrow D$ such that $\delta_{\omega} \circ f=\gamma_{\omega}$. 
Remark 3.6. Clearly, every morphism of coalgebras $f:(C, \gamma) \rightarrow(D, \delta) \in$ $\operatorname{Coalg}(T)$ is also a morphism $f \in \operatorname{Beh}_{\omega}(T)$. We hence obtain a functorial inclusion Coalg $(T) \rightarrow \operatorname{Beh}_{\omega}(T)$. In order to explain the relationship of $\operatorname{Beh}_{\omega}(T)$ to Coalg $(T)$ consider the following categories

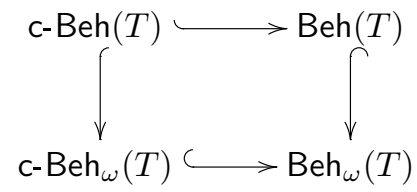

which all have coalgebras as objects and morphisms as follows. $f:(C, \gamma) \rightarrow$ $(D, \delta)$ is a $\operatorname{Beh}(T)$-morphism iff $\gamma_{n}(c)=\delta_{n}(f(c))$ for all ordinals $n$ and all $c \in C$. The definitions of $\mathrm{c}-\operatorname{Beh}(T)$ and $c-\operatorname{Beh}_{\omega}(T)$ follow the same idea, but take colourings into account: $f:(C, \gamma) \rightarrow(D, \delta)$ is a c-Beh ${ }_{\omega}(T)$-morphism iff $f$ is a $\operatorname{Beh}_{\omega}(T \times X)$-morphism $(C,\langle\gamma, v \circ f\rangle) \rightarrow(D,\langle\delta, v\rangle)$ for all $X \in$ Set and $v: D \rightarrow X$.

If Coalg $(T)$ has cofree coalgebras then c-Beh $(T)=\operatorname{Coalg}(T)$. If $T$ is finitary (ie. $\omega$-accessible) then $\operatorname{Beh}_{\omega}(T)=\operatorname{Beh}(T)$ and $c-B e h \omega(T)=\mathrm{c}-\operatorname{Beh}(T)$. Whether the converse holds, that is, whether c-Beh $\omega(T)=\mathrm{c}-\operatorname{Beh}(T)$ implies that $T$ is finitary is an open question.

We conclude the section by collecting some simple properties of $\operatorname{Beh}_{\omega}(T)$, all of which are also present in Coalg $(T)$ :

Proposition 3.7. Suppose $U: \operatorname{Beh}_{\omega}(T) \rightarrow$ Set is the forgetful functor.

1. $\operatorname{Beh}_{\omega}(T) \hookrightarrow \operatorname{Coalg}(T)$ preserves and reflects coproducts.

2. Injective and surjective morphisms form a factorisation system for $\operatorname{Beh}_{\omega}(T)$. In particular, every morphism $f \in \operatorname{Beh}_{\omega}(T)$ factors as $f=m \circ$ e with $U m$ mono, Ue epi.

Proof. The claim on coproducts is immediate. Concerning factorisations, let $f:(C, \gamma) \rightarrow(D, \delta)$ be a morphism in $\operatorname{Beh}_{\omega}(T)$ and $C \stackrel{e}{\rightarrow} I \stackrel{m}{\rightarrow} D$ be its epi-mono factorisation in Set. Choose $h$ with $e \circ h=i d_{I}$ and define $\iota: I \rightarrow T I$ as Teo $\gamma \circ h$. Assuming $\iota_{n} \circ e=\gamma_{n}$, we verify $\iota_{n+1} \circ e=T \iota_{n} \circ \iota \circ e=\gamma_{n+1} \circ h \circ e=\delta_{n+1} \circ f \circ h \circ e=$ $\delta_{n+1} \circ f=\gamma_{n+1}$, showing that $e:(C, \gamma) \rightarrow(I, \iota)$ is a morphism, and hence also $m$. We have seen that factorisations exist in $\operatorname{Beh}_{\omega}(T)$. The remaining conditions on a factorisation system (see e.g. [1]) are easy to check.

\section{$4 \quad$ Final and Quasi-Canonical Models}

Reasoning about behaviours, the final coalgebra plays a central role because, given the unique coalgebra morphism $!_{C}: C \rightarrow Z$ from a coalgebra $C$ into the final coalgebra $Z$, for every element $c$ of (the carrier of) $C$, we can consider $!_{C}(c)$ as the behaviour of $c$. Similarly, final objects of $\operatorname{Beh}_{\omega}(T)$ (cf. Definition 3.5) consist of the finite behaviours. This section shows, that $\operatorname{Beh}_{\omega}(T)$ always has 
a final object, which generalises the canonical model construction from Kripke models to coalgebras.

\subsection{Final Objects in $\operatorname{Beh}_{\omega}(T)$}

A final object of $\operatorname{Beh}_{\omega}(T)$ should "realise" all $n$-step behaviours, $n<\omega$. Accordingly, the carrier of a final object in $\operatorname{Beh}_{\omega}(T)$ will be a subset of $T^{\omega} 1$.

Recall that, given any structure $(C, \gamma)$, we write $\gamma_{\omega}$ for the unique mediating map $\gamma_{\omega}: C \rightarrow T^{\omega} 1$. That is, all $\omega$-step behaviours appear as some $\gamma_{\omega}(c)$ in $T^{\omega} 1$. On the other hand, it may happen that not every point $t \in T^{\omega} 1$ can be presented as $t=\gamma_{\omega}(c)$ by some structure $(C, \gamma)$ and some $c \in C$. Consider for example the finite powerset functor $T=\mathcal{P}_{\omega}$. Worrell [31] shows, that for the final $T$-coalgebra $(Z, \zeta)$ the morphism $\zeta_{\omega}: Z \rightarrow T^{\omega} 1$ is (injective but) not surjective.

Hence we construct the carrier of the coalgebra final in $\operatorname{Beh}_{\omega}(T)$ by collecting all $t \in T^{\omega} 1$ which can be "realised" by some structure, i.e. for which there are $(C, \gamma) \in \operatorname{Coalg}(T)$ and $c \in C$ such that $\gamma_{\omega}(c)=t$. It then remains to find an appropriate coalgebra structure.

Throughout, we fix the set $K$ of "realisable" elements $t \in T^{\omega} 1$, which is given by

$$
K=\left\{t \in T^{\omega} 1 \mid \exists(C, \gamma) \in \operatorname{Coalg}(T) . \exists c \in C \cdot \gamma_{\omega}(c)=t\right\} .
$$

For each $k \in K$, we can now choose $\left(C^{k}, \gamma^{k}\right) \in \operatorname{Coalg}(T)$ and $c^{k} \in C^{k}$ such that $\gamma_{\omega}^{k}\left(c_{k}\right)=k$. Note that $K$ is a set, which enables us to consider

$$
(C, \gamma)=\coprod_{k \in K}\left(C^{k}, \gamma^{k}\right)
$$

where the coproduct is taken in $\operatorname{Coalg}(T)$. Denoting the coproduct injections by $i n_{k}: C_{k} \rightarrow C$ (which, by the construction of coproducts in Coalg $(T)$ are also coproduct injections in the category of sets), we are ready to note:

Lemma 4.1. $\gamma_{\omega} \circ i n_{k}(c)=\gamma_{\omega}^{k}(c)$ for all $k \in K$ and $c \in C_{k}$.

Proof. Since $\gamma_{\omega}^{k}$ is the unique mediating map into the limiting cone with vertex $T^{\omega} 1$, it suffices to prove that $\gamma_{n} \circ i n_{k}(c)=\gamma_{n}^{k}(c)$ for all $n<\omega$. For $n=0$, this is obvious. For the induction step we calculate $\gamma_{n+1} \circ i n_{k}(c)=T \gamma_{n} \circ \gamma \circ i n_{k}(c)=$ $T \gamma_{n} \circ \operatorname{Tin}_{k} \circ \gamma^{k}(c)=T \gamma_{n}^{k} \circ \gamma^{k}(c)=\gamma_{n+1}^{k}(c)$.

We obtain the following immediate corollary:

Corollary 4.2. For all $k \in K$ there exists $c \in C$ with $\gamma_{\omega}(c)=k$.

In other words, $\gamma_{\omega}$ factors through $K$ as $\gamma_{\omega}=m \circ e, m$ injective, $e$ surjective. 
Now consider the diagram

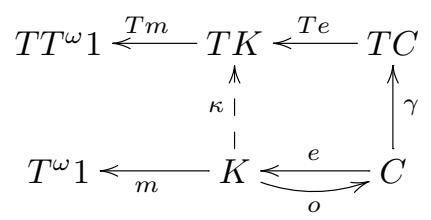

where $o$ is any one-sided inverse of $e$, i.e. $e \circ o=i d_{K}$, the existence of which is guaranteed by $e$ being a surjection. We let

$$
\kappa=T e \circ \gamma \circ o .
$$

Note that $\kappa: K \rightarrow T K$ makes $K$ into a $T$-coalgebra. Denoting the limit projections by $p_{n}^{\omega}: T^{\omega} 1 \rightarrow T^{n} 1$, we obtain

Lemma 4.3. For all $n<\omega, \kappa_{n}=p_{n}^{\omega} \circ m$, hence $m=\kappa_{\omega}$.

Proof. We proceed by induction on $n$, where the case $n=0$ is evident. We calculate $\kappa_{n+1}=T \kappa_{n} \circ \kappa=T\left(p_{n}^{\omega} \circ m\right) \circ T e \circ \gamma \circ o=T p_{n}^{\omega} \circ T(m \circ e) \circ \gamma \circ o=$ $T p_{n}^{\omega} \circ T \gamma_{\omega} \circ \gamma \circ O=T \gamma_{n} \circ \gamma \circ O=\gamma_{n+1} \circ 0=p_{n+1}^{\omega} \circ \gamma_{\omega} \circ O=p_{n+1}^{\omega} \circ m \circ e \circ o=p_{n+1}^{\omega} \circ m$ for the induction step, as desired.

The proof of the main theorem of this section is now straightforward.

Theorem 4.4. $\operatorname{Beh}_{\omega}(T)$ has a final object.

Proof. We show that $(K, \kappa)$, as constructed above, is final in $\operatorname{Beh}_{\omega}(T)$. Take any object $(D, \delta) \in \operatorname{Beh}_{\omega}(T)$. Consider the mapping $\delta_{\omega}: D \rightarrow T^{\omega} 1$, which is the unique mediating map between the cones $\left(D,\left(\delta_{n}\right)_{n<\omega}\right)$ and $\left(T^{\omega} 1,\left(p_{n}^{\omega}\right)_{n<\omega}\right)$. By construction, $\delta_{\omega}$ factors as $\delta_{\omega}=m \circ h$ where $m: K \rightarrow T^{\omega} 1$ is as above. By Lemma 4.3

$$
\delta_{\omega}=\kappa_{\omega} \circ h
$$

which implies that $h$ is a $\operatorname{Beh}_{\omega}(T)$-morphism. $h$ is unique since $\kappa_{\omega}$ is injective.

Note that final objects in $\operatorname{Beh}_{\omega}(T)$ are not determined uniquely up to Coalg $(T)$ isomorphism. In case that $p_{\omega}^{\omega+1}: T T^{\omega} 1 \rightarrow T^{\omega} 1$ is surjective ${ }^{1}$, final objects of $\operatorname{Beh}_{\omega}(T)$ arise as $\left(T^{\omega} 1, \theta\right)$ where $\theta$ is a right inverse of $p_{\omega}^{\omega+1}$.

Corollary 4.5. Assume that $p_{\omega}^{\omega+1}$ is surjective. An object is final in $\operatorname{Beh}_{\omega}(T)$ iff it is isomorphic in $\operatorname{Coalg}(T)$ to some $\left(T^{\omega} 1, \theta\right)$ with $p^{\omega+1} \circ \theta=i d_{T^{\omega}{ }_{1}}$

Proof. 'if': To show that $\left(T^{\omega} 1, \theta\right)$ is final, it suffices to observe that $\theta_{\omega}=i d_{T^{\omega}}$. This follows from $\theta_{n}=p_{n}^{\omega}, n<\omega$, the inductive case being $\theta_{n+1}=T\left(\theta_{n}\right) \circ \theta=$ $T\left(p_{n}^{\omega}\right) \circ \theta=p_{n+1}^{\omega} \circ p_{n}^{\omega+1} \circ \theta=p_{n+1}^{\omega}$.

\footnotetext{
${ }^{1}$ Which is the case for all examples in this paper with the exception of $T=\mathcal{P}_{\omega}$. A sufficient condition for $p_{\omega}^{\omega+1}$ to be surjective is that $T$ weakly preserves limits of $\omega^{\text {op}}$-chains.
} 
'only if': Let $(C, \gamma)$ be final in $\operatorname{Beh}_{\omega}(T)$. Consider a final object $(K, \kappa)$ as constructed in the proof of the theorem. Let $f:(C, \gamma) \rightarrow(K, \kappa)$ be the unique morphism. In particular, $f$ is iso and $\kappa_{\omega} \circ f=\gamma_{\omega}$. Since $\kappa_{\omega}$ is injective, $\gamma_{\omega}$ is as well. Since, by Proposition 1.4(ii), $\gamma_{\omega}=p_{\omega}^{\omega+1} \circ T\left(\gamma_{\omega}\right) \circ \gamma, \gamma_{\omega}$ is also surjective, hence iso. Now define $\theta=T\left(\gamma_{\omega}\right) \circ \gamma \circ \gamma_{\omega}^{-1}$.

We conclude with the useful observation that all $t \in T^{n} 1, n<\omega$, are realised as $n$-step behaviours in the final $\operatorname{Beh}_{\omega}(T)$-coalgebra. We first note that every element of an 'approximant' $T^{n} 1$ is realised by a coalgebra.

Proposition 4.6. Let $f$ be any mapping $1 \rightarrow T 1$ and $\left(C^{n}, \gamma^{n}\right)=\left(T^{n} 1, T^{n} f\right)$. Then $\gamma_{n}^{n}=i d_{C^{n}}$.

As an immediate corollary we obtain that the maps $\kappa_{n}$ are surjections:

Corollary 4.7. Suppose $(K, \kappa)$ is final in $\operatorname{Beh}_{\omega}(T)$ and $n<\omega$. Then $\kappa_{n}$ is a surjection.

Proof. Let $\left(C^{n}, \gamma^{n}\right)$ be given as in the above proposition. If $x \in T^{n} 1$, we have $x=\gamma_{n}^{n}(x)=\kappa_{n} \circ !(x)$, where ! : $\left(C^{n}, \gamma^{n}\right) \rightarrow(K, \kappa)$ is the map given by finality.

\subsection{The Canonical Model}

Let $M$ be the functor $\mathcal{P} \times \mathcal{P}$ Prop, Prop a countably infinite set.

The canonical model (see for example [4, 7]) for the modal logic $\mathcal{M L}$ is the $M$-coalgebra $\left(L,\left\langle\lambda_{R}, \lambda_{V}\right\rangle\right)$

$$
\begin{array}{ll}
L & \{\Phi \subseteq \mathcal{M L}: \Phi \text { is maximally consistent }\} \\
\lambda_{R}: L \rightarrow \mathcal{P} L & \Phi \mapsto\{\Psi: \psi \in \Psi \Rightarrow \diamond \psi \in \Phi\} \\
\lambda_{V}: L \rightarrow \mathcal{P} \text { Prop } & \Phi \mapsto \Phi \cap \text { Prop }
\end{array}
$$

The canonical model is final in the category $\mathrm{Th}_{\mathcal{M L}}$ which has $M$-coalgebras as objects and morphisms $f:(C, \gamma) \rightarrow(D, \delta)$ are functions $f: C \rightarrow D$ such that for all $c \in C, c$ and $f(c)$ have the same modal theory.

Proposition 4.8. $\operatorname{Beh}_{\omega}(M) \cong \mathrm{Th}_{\mathcal{M L}}$.

Proof. We have to show that for any coalgebras $(C, \gamma),(D, \delta)$ and any function $f: C \rightarrow D$,

$$
\delta_{\omega} \circ f(c)=\gamma_{\omega}(c) \Leftrightarrow \operatorname{Th}(c)=\operatorname{Th}(f(c)),
$$

which is equivalent to $\left[\forall n<\omega . \delta_{n} \circ f(c)=\gamma_{n}(c)\right] \Leftrightarrow[\forall n<\omega . \forall \varphi \in \mathcal{M L}$. $\operatorname{rank}(\varphi)=n \Rightarrow(c \models \varphi \Leftrightarrow f(c) \models \varphi)]$ which can be shown using induction on $n$.

It follows that the canonical model is final in $\operatorname{Beh}_{\omega}(M)$. We show now that, conversely, every final object in $\operatorname{Beh}_{\omega}(M)$ satisfies the so-called truth-lemma which is the main property of the canonical model. 
Definition 4.9. A $M$-coalgebra $(L, \lambda)$ is called a quasi-canonical model if $L$ is the set of maximal consistent sets of formulae and

$$
(L, \lambda), \Phi \models \varphi \Longleftrightarrow \varphi \in \Phi .
$$

for all $\Phi \in L$.

The canonical model is quasi-canonical. In fact, it is Property (2) which makes the canonical model useful. Hence, any quasi-canonical model can serve the same purpose as the canonical model. The following gives a syntax-free characterisation of the quasi-canonical models. Moreover, the theorem shows that the quasi-canonical models are precisely - up to isomorphism of coalgebras - the final coalgebras constructed in the previous subsection.

Theorem 4.10. Suppose $\mathrm{C}$ is a T-coalgebra. Then $\mathrm{C}$ is final in $\mathrm{Beh}_{\omega}(T)$ iff $\mathrm{C}$ is Coalg $(T)$-isomorphic to a quasi-canonical model.

Proof. First, every quasi-canonical model is easily seen to be final in $T_{\mathcal{M L}}$ and hence, by Proposition 4.8, final in $\operatorname{Beh}_{\omega}(M)$. Now suppose $(C, \gamma)$ is final in $\operatorname{Beh}_{\omega}(T)$. Since the canonical model $\left(L,\left\langle\lambda_{R}, \lambda_{V}\right\rangle\right)$ is also final in $\operatorname{Beh}_{\omega}(T)$, the map $f: C \rightarrow L, c \mapsto\{\varphi \in \mathcal{M L}|c|=\varphi\}$ is a bijection. Let $\gamma^{\prime}=T f^{-1} \circ \gamma \circ f^{-1}$. Then $(C, \gamma) \cong\left(L, \gamma^{\prime}\right) \in \operatorname{Coalg}(T)$. It remains to show the truth lemma for $\left(L, \gamma^{\prime}\right):\left(L, \gamma^{\prime}\right), \Phi \models \varphi \Leftrightarrow(C, \gamma), f^{-1}(\Phi) \models \varphi \Leftrightarrow \varphi \in f\left(f^{-1}(\Phi)\right) \Leftrightarrow \varphi \in$ $\Phi$.

Since for $M X=\mathcal{P} X \times \mathcal{P}$ Prop the projection $p_{\omega}^{\omega+1}$ is easily seen to be surjective, Corollary 4.5 shows, that the choice of transition relation on a quasicanonical model corresponds to the choice of right inverse of the projection $p_{\omega}^{\omega+1}$.

Corollary 4.11. There is a 1-1 correspondence between the set of quasi-canonical models and the set of right inverses of $p_{\omega}^{\omega+1}$.

\section{Logics of Finite Rank and Logics of Rank $\omega$}

In Section 3, we introduced a notion of finite step equivalence between elements of coalgebras. This section starts the investigation of logics whose formulas are invariant under finite step equivalence. Since we do not want to commit ourselves to a particular syntax, we assume that a logic $\mathcal{L}$ for $T$-coalgebras already comes with an interpretation function $\llbracket \cdot \rrbracket_{(C, \gamma)}: \mathcal{L} \rightarrow \mathcal{P}(C)$ for every $T$-coalgebra $(C, \gamma)$, which maps a formula $\varphi \in \mathcal{L}$ to the set $\llbracket \varphi \rrbracket \subseteq C$ of states which satisfy $\varphi$.

Definition 5.1. An abstract logic for $T$-coalgebras is a pair $(\mathcal{L}, \llbracket \cdot \rrbracket)$ where

- $\mathcal{L}$ is the set of formulas and

- $\llbracket \cdot \rrbracket$ is a family of mappings $\llbracket \cdot \rrbracket_{(C, \gamma)}: \mathcal{L} \rightarrow \mathcal{P}(C)$, indexed by the $T$ coalgebras, 
such that $\mathcal{L}$ has (classical) negations and conjunctions, which are interpreted as complement and intersection, respectively.

For $\varphi \in \mathcal{L}$ and $t \subseteq T^{n} 1$, we say that $t$ represents $\varphi$ iff $\llbracket \varphi \rrbracket_{(C, \gamma)}=\gamma_{n}^{-1}(t)$ for all $T$-coalgebras $(C, \gamma)$. In this case, we say that $\varphi$ has rank $n$.

$(\mathcal{L}, \llbracket \cdot \rrbracket)$ is of finite rank iff every $\varphi \in \mathcal{L}$ has finite rank. We say that $(\mathcal{L}, \llbracket \cdot \rrbracket)$ has $\operatorname{rank} \omega$, if every $\varphi \in \mathcal{L}$ has rank $\omega$.

If $(C, \gamma)$ is a $T$-coalgebra, $c \in C$ and $\varphi \in \mathcal{L}$, we often write $c \models_{(C, \gamma)} \varphi$ for $c \in \llbracket \varphi \rrbracket_{(C, \gamma)}$ and drop the subscripts if there is no danger of confusion.

Example 5.2. Propositional modal logic is our prime example of a logic of finite rank (Proposition 2.1). A logic of finite rank is also of rank $\omega$. Linear temporal logic is an example of a logic of rank $\omega$ which is not of finite rank (Example 2.2).

In case that the final coalgebra $(Z, \zeta)$ exists, we can represent any logic $\mathcal{L}$ whose formulae are invariant under behavioural equivalence by $\llbracket \cdot \rrbracket_{(Z, \zeta)}: \mathcal{L} \rightarrow$ $\mathcal{P} Z$, the $\llbracket \cdot \rrbracket_{(C, \gamma)}$ being determined by $\llbracket \cdot \rrbracket_{(C, \gamma)}=!_{(C, \gamma)}^{-1} \circ \llbracket \cdot \rrbracket_{(Z, \zeta)}$ where $!_{(C, \gamma)}$ : $(C, \gamma) \rightarrow(Z, \zeta)$ is given by finality in Coalg $(T)$. Similarly, a logic $\mathcal{L}$ of rank $\omega$ can be represented by $\llbracket \cdot \rrbracket_{(K, \kappa)}$ :

Proposition 5.3. Suppose $(\mathcal{L}, \llbracket \cdot \rrbracket)$ is of rank $\omega$ and $(K, \kappa)$ is final in $\operatorname{Beh}_{\omega}(T)$. Then $\llbracket \varphi \rrbracket_{(C, \gamma)}=!_{C}^{-1}\left(\llbracket \varphi \rrbracket_{(K, \kappa)}\right)$ for all $\varphi \in \mathcal{L}$ and all T-coalgebras $(C, \gamma)$, where $!_{C}:(C, \gamma) \rightarrow(K, \kappa)$ is the unique morphism given by finality.

Proof. Let $\varphi \in \mathcal{L}$. By assumption, there is $t \subseteq T^{\omega} 1$ such that $\llbracket \varphi \rrbracket_{(C, \gamma)}=\gamma_{\omega}^{-1}(t)$ for all $T$-coalgebras $(C, \gamma)$. Since $!_{C}$ is a morphism of $\operatorname{Beh}_{\omega}(T)$, we have $\gamma_{\omega}=$ $\kappa_{\omega} \circ !_{C}$. Thus $\llbracket \varphi \rrbracket_{(C, \gamma)}=\gamma_{\omega}^{-1}(t)=!_{C}^{-1} \circ \kappa_{\omega}^{-1}(t)=!_{C}^{-1}\left(\llbracket \varphi \rrbracket_{(K, \kappa)}\right)$.

We conclude that logics of rank $\omega$ are precisely those logics, whose formulas are invariant under finite step equivalence:

Corollary 5.4. A logic is of rank $\omega$ iff its formulas are invariant under finite step equivalence, i.e., iff $c \sim_{n} d \Rightarrow(c \models \varphi \Leftrightarrow d \models \varphi)$ for all $\varphi \in \mathcal{L}$, $(C, \gamma),(D, \delta) \in \operatorname{Coalg}(T), c \in C, d \in D, n<\omega$.

Proof. Follows from the above proposition and the observation that, given $T$ coalgebras $(C, \gamma),(D, \delta)$ and $(c, d) \in C \times D$, we have $c \sim_{\omega} d$ iff $!_{C}(c)=!_{D}(d)$, where $!_{C}$ and $!_{D}$ are the unique morphisms into the final object of $\operatorname{Beh}_{\omega}(T)$.

In the general case of (infinitary) logics we called a logic fully expressive if all subsets of the final coalgebra can be presented as the semantics of a formula. In our context the following expressiveness condition is suitable.

Definition 5.5. We call a logic $\mathcal{L}$ finite step expressive iff for all $n<\omega$ and all $t \subseteq T^{n} 1$ there is $\varphi \in \mathcal{L}$ such that $t$ represents $\varphi$. 


\section{The Topology Induced by a Logic}

We have seen in Section 5 , that logics $\mathcal{L}$ whose formulas $\varphi \in \mathcal{L}$ are invariant under finite step equivalence can be represented by $\llbracket \cdot \rrbracket_{\mathrm{K}}: \mathcal{L} \rightarrow \mathcal{P} K$ where $\mathrm{K}=(K, \kappa)$ is the final object of $\operatorname{Beh}_{\omega}(T)$. In general, one can not expect of a logic of finite rank that all subsets of $K$ are expressible in the logic. In this section, we study logics in terms of the topology generated by the (denotations of) the formulae of $\mathcal{L}$; introductory material on the relation between logic and topology can be found in $[19,30]$. For the rest of the paper, we assume that $(\mathcal{L}, \llbracket \cdot \rrbracket)$ is an abstract logic (Definition 5.1).

Definition 6.1 (Topologies $\tau_{C}$ ). Suppose $(C, \gamma)$ is a $T$-coalgebra. The topology $\tau_{C}$ on $C$ is generated by the basis $\left\{\llbracket \varphi \rrbracket_{(C, \gamma)} \mid \varphi \in \mathcal{L}\right\}$.

Remark 6.2. Suppose $f:(C, \gamma) \rightarrow(D, \delta) \in \operatorname{Beh}_{\omega}(T)$. If $\mathcal{L}$ is of rank $\omega$, the semantics of formulae is stable under $\operatorname{Beh}_{\omega}(T)$-morphisms (Corollary 5.4), hence $f:\left(C, \tau_{C}\right) \rightarrow\left(D, \tau_{D}\right)$ is continuous. Since every morphism of coalgebras qualifies as a $\operatorname{Beh}_{\omega}(T)$-morphism, we have a chain of functors $\operatorname{Coalg}(T) \rightarrow$ $\operatorname{Beh}_{\omega}(T) \rightarrow$ Top, where Top is the category of topological spaces.

By definition, every formula of a logic of finite rank can be represented as subset $t \subseteq T^{n} 1$ for some $n<\omega$. If the approximants $T^{n} 1$ are finite it is natural to assume that all subsets of $T^{n} 1$ can be expressed by a formula, that is, that $\mathcal{L}$ is finite step expressive (cf. Definition 5.5). Since this is not the case in general (see eg. propositional modal logic as in Section 2.1), we introduce topologies also on the approximants $T^{n} 1$.

Definition 6.3 (Topologies $\tau_{C}^{\omega}$ ). For $n<\omega$, the topology $\tau_{n}$ on $T^{n} 1$ is given by the basis $\left\{t \subseteq T^{n} 1 \mid \exists \varphi \in \mathcal{L}\right.$.t represents $\left.\varphi\right\}$. If $(C, \gamma)$ is a $T$-coalgebra, the topology $\tau_{C}^{\omega}$ on $C$ is given by the basis $\left\{\gamma_{n}^{-1}(U) \mid U \in \tau_{n}, n<\omega\right\}$.

The topology on the approximants would not be worth its salt if it would not turn the connecting morphisms $p_{m}^{n}: T^{n} 1 \rightarrow T^{m} 1$ into continuous functions:

Remark 6.4. Suppose $m \leq n<\omega$ and $t \subseteq T^{n} 1$ represents a formula $\varphi$ of $\mathcal{L}$ (that is, $t$ is a basic open of $\left.\left(T^{n} 1, \tau_{n}\right)\right)$. Then $\left(p_{m}^{n}\right)^{-1}(t)$ also represents $\varphi$, showing that $p_{m}^{n}$ is continuous.

The following easy proposition is useful in that it allows to compute the topologies $\tau_{C}$ via the topologies on the approximants $T^{n} 1$.

Proposition 6.5. Let $\mathcal{L}$ be a logic of finite rank and $(C, \gamma)$ a coalgebra. Then the topologies $\tau_{C}^{\omega}$ and $\tau_{C}$ coincide.

The converse of the proposition only holds if $A$ is compact. Before we turn to compactness issues, we discuss an important special case:

Definition 6.6 (Cantor space topology). In case that $\mathcal{L}$ is finite step expressive, that is, in case the topologies $\tau_{n}$ are discrete, we call $\tau_{C}^{\omega}$ the Cantor space topology. 
The terminology is motivated by the following example.

Example 6.7. Suppose $T X=2 \times X$, where $2=\{0,1\}$. Consider the (final) $T$-coalgebra $(C, \gamma)$ with $C=2^{\omega}=\{f: \omega \rightarrow 2\}$ and $\gamma(f)=(f(0), \lambda n . f(n+1))$. Then $\left(C, \tau_{C}\right)$ is homeomorphic to the Cantor discontinuum $\mathbb{C}$ (also known as middle-third set, see e.g. [11]) via the mapping $2^{\omega} \rightarrow \mathbb{C}, f \mapsto \sum_{i=0}^{\infty} \frac{2}{3^{i+1}} \cdot f(i)$.

Remark 6.8. Let $(C, \gamma) \in \operatorname{Coalg}(T)$ and let, for $c_{0}, c_{1} \in C, d_{C}\left(c_{0}, c_{1}\right)=$ $\inf \left\{2^{-n}: \forall k<n \cdot \gamma_{k}\left(c_{0}\right)=\gamma_{k}\left(c_{1}\right)\right\}$. Then $d_{C}$ is a pseudo-ultrametric on $C$, and $d_{C}$ is a ultrametric if $\gamma_{\omega}: C \rightarrow T^{\omega} 1$ is injective. The Cantor space topology $\tau_{C}$ coincides with the topology induced by $d_{C}$, as studied in $[3,32]$.

In the remainder of the section we relate topological and logical notions. All of the results below are consequences of the following observations

- the basic opens are precisely the subsets expressible by single formulae,

- the basic opens are closed under complements and finite unions,

where the second point is due to the requirement that our logics are closed under boolean operators, see Definition 5.1.

We shall often require our topologies to be compact and Hausddorff. ${ }^{2}$ The relationship of these properties to logical issues is the content of the following proposition:Recall the notation $k \models \varphi \Leftrightarrow k \in \llbracket \varphi \rrbracket$ and $\Phi=\varphi \Leftrightarrow \bigcap\{\llbracket \varphi \rrbracket \mid \varphi \in$ $\Phi\} \subseteq \llbracket \varphi \rrbracket$.

Proposition 6.9. Suppose $(K, \kappa)$ is final in $\operatorname{Beh}_{\omega} T$.

1. $K$ is Hausdorff iff for all distinct $k_{1}, k_{2}$ in $K$ there is $\varphi \in \mathcal{L}$ such that $k_{1} \models \varphi$ and $k_{2} \models \neg \varphi$.

2. $K$ is compact iff for all $\Phi \subseteq \mathcal{L}, \varphi \in \mathcal{L}$ with $\Phi \models \varphi$ there is a finite subset $\Phi^{\prime} \subseteq \Phi$ with $\Phi^{\prime} \models \varphi$.

Logically speaking, $K$ is Hausdorff iff $\mathcal{L}$ is expressive in the sense that every pair of different states can be separated by a formula. The last property says that if $\varphi$ is a consequence of a set $\Phi$ of formulas, there is a finite subset $\Phi^{\prime} \subseteq \Phi$ such that $\Phi^{\prime}$ already forces the validity of $\varphi$. For finitary logics with a sound and complete axiomatisation, this is always the case, since a proof of $\varphi$ from $\Phi$ can only use finitely many premises. Since we study logics without making any commitment to a particular syntax, this property is not guaranteed, and we have to require it for a number of results in the sequel.

The following are easy consequences of the definition of the topologies as generated by the semantics of modal formulas.

Proposition 6.10. Let $(C, \gamma) \in \operatorname{Coalg}(T)$. A subset of $C$ is definable by a set of formulae iff it is closed w.r.t. $\tau_{C}$.

\footnotetext{
${ }^{2} \mathrm{~A}$ set is compact iff any open cover has a finite subcover. This is sometimes called quasicompact. A space $(X, \tau)$ is Hausdorff iff $\forall x, y \in X . x \neq y \Rightarrow \exists U, V \in \tau . x \in U \wedge y \in$ $V \wedge U \cap V=\emptyset$.
} 
Proof. " $\Rightarrow$ ", since a set expressible by a formula is closed and the intersection of closed sets is closed. " $\Leftarrow$ ", since a set is closed only if there are basic opens $O_{i}, i \in I$, such that $S=\bigcap\left\{C \backslash O_{i} \mid i \in I\right\}$ and the complement of a basic open is expressible by a formula.

Proposition 6.11. Suppose $(C, \gamma) \in \operatorname{Coalg}(T)$ and $\left(C, \tau_{C}\right)$ compact. Then a subset $S \subseteq C$ is expressible by a single formula in $\mathcal{L}$ iff $S$ is clopen.

Proof. " $\Rightarrow$ " is immediate. For " $\Leftarrow "$ ", let $S$ be clopen. Since $S$ is open, there are basic opens $O_{i}, i \in I$, such that $S=\bigcup\left\{O_{i} \mid \in I\right\}$. S is compact, since $S$ is closed and $C$ is compact. Hence there is a finite subcover. Since the basic opens are closed under finite unions, $S$ is a basic open.

From a logical point of view, compactness corresponds to finiteness of proofs and is therefore not an issue for finitary logics, which have a sound and complete axiomatisation. However, there are models which are not compact:

Example 6.12. Let $T X=D \times X$ and consider the final coalgebra $(Z, \zeta)$ given by $Z=D^{\omega}$.

1. $(Z, \zeta)$ is compact in the Cantor space topology iff $D$ is finite.

2. Suppose $D=\{a, b\}$. Then examples of non-compact coalgebras are given by the carriers $Z \backslash\left\{b^{\omega}\right\}$ and $\left\{s \cdot a^{\omega}: s \in\{a, b\}^{*}\right\}$ (and inheriting the structure from $\zeta$ ).

Example 6.13. Let $T X=\{a, b\} \times X+1$ and consider the final coalgebra $(Z, \zeta)$ with $Z=\{a, b\}^{*} \cup\{a, b\}^{\omega}$. Then $Z$ is compact in the Cantor space topology (since the limit of compact Hausdorff spaces is compact Hausdorff, see $[5], 3.2 .13)$ and $\{a, b\}^{*}$ is not compact. The topology on $Z$ is as follows. A subset of $Z$ is open iff it is a subset of $\{a, b\}^{*}$ or of the form $V \cdot\left(\{a, b\}^{*}+\{a, b\}^{\omega}\right)$ for some $V \subseteq\{a, b\}^{*}$. In particular, every open cover of $\{a, b\}^{\omega}$ also covers $\{a, b\}^{*}$.

Another example is given by $T X=\mathcal{P}_{\omega}(X)$ for which the final $T$-coalgebra is not compact. This can most easily be seen using the next proposition. Given a $\operatorname{logic} \mathcal{L}$ for $T$, we call a $T$-coalgebra $(C, \gamma)$ logically compact, if every set, which is finitely satisfiable in $(C, \gamma)$ (that is, for every finite subset $\Phi^{\prime} \subseteq \Phi$ there exists $c \in C$ such that $\left.c=\Phi^{\prime}\right)$ is satisfiable in $(C, \gamma)$ (i.e. there exists $c \in C$ such that $c=\Phi)$. We are now ready to prove

Proposition 6.14. Let $(C, \gamma) \in \operatorname{Coalg}(T)$ and $\mathcal{L}$ a logic for $T$. Then $(C, \gamma)$ is logically compact iff $\left(C, \tau_{C}\right)$ is a compact topological space.

Proof. We use that $C$ is compact iff every set $\mathcal{S} \subseteq \mathcal{P}(C)$ of closed subsets that has the finite intersection property ${ }^{3}$ has non-empty intersection.

Assume that $(C, \gamma)$ is logically compact and that $\mathcal{S} \subseteq \mathcal{P}(C)$ is a set of closed sets having the finite intersection property. Every set $S \in \mathcal{S}$ is definable by some $\Phi_{S} \subseteq \mathcal{L}$ (Proposition 6.10). It follows from $\mathcal{S}$ having the finite intersection

\footnotetext{
${ }^{3} \mathcal{S}$ has the finite intersection property iff $\cap \mathcal{S}^{\prime}$ is non-empty for all finite $\mathcal{S}^{\prime} \subseteq \mathcal{S}$.
} 
property that $\bigcup\left\{\Phi_{S} \mid S \in \mathcal{S}\right\}$ is finitely satisfiable and hence satisfiable. That is, there exists $c \in C$ such that $c \models \bigcup\left\{\Phi_{S} \mid S \in \mathcal{S}\right\}$ which implies $c \in \bigcap \mathcal{S}$.

Now assume $\left(C, \tau_{C}\right)$ is topologically compact and consider a set $\Phi \subseteq \mathcal{L}$ which is finitely satisfiable. Since $\llbracket \varphi \rrbracket \subseteq C$ is closed by Proposition 6.10 , the set $\{\llbracket \varphi \rrbracket: \varphi \in \Phi\}$ is a set of closed sets having the finite intersection property. By compactness of $\left(C, \tau_{C}\right)$, there exits $c \in \bigcap\{\llbracket \varphi \rrbracket \mid \varphi \in \Phi\}$, that is, $c \models \Phi$.

Example 6.15. For finitely branching Kripke structures, ie. $T=\mathcal{P}_{\omega}$, it is not difficult to write down formulae $\varphi_{n}$ which force any point satisfying $\varphi_{n}$ to have at least $n$ successors. The set $\Phi=\left\{\varphi_{n} \mid n<\omega\right\}$ is then finitely satisfiable, but not satisfiable by a $\mathcal{P}_{\omega}$-coalgebra.

\section{Compactness for Logics of Rank $\omega$}

In (standard) modal logic, compactness is not an issue, since it is inherited from the compactness of first order logic via van Benthem's standard translation $[29,4]$. Generalising to coalgebras, the standard translation is no longer available. Moreover, compactness fails for example in the case of image-finite Kripke models (i.e. $T=\mathcal{P}_{\omega}$, cf. Example 6.15). Hence we are drawn to investigate sufficient and necessary conditions for the compactness theorem to hold.

We say that a set $\Phi \subseteq \mathcal{L}$ is satisfiable, if there exists a $T$-coalgebra $(C, \gamma)$ such that $\Phi$ is satisfiable in $(C, \gamma)$. We call $\Phi$ finitely satisfiable, if every finite subset of $\Phi$ is satisfiable. Finally, a logic $\mathcal{L}$ is compact, if every finitely satisfiable set of formulas is satisfiable. Using this terminology, we are in the position to present the first version of the compactness theorem.

Theorem 7.1. Suppose $\mathcal{L}$ is of rank $\omega$. Then $\mathcal{L}$ is compact iff $\operatorname{Beh}_{\omega}(T)$ has a compact final object.

Proof. 'only if': By Theorem 4.4 there exists a final object $(K, \kappa) \in \operatorname{Beh}_{\omega}(T)$. We show that $(K, \kappa)$ is logically compact, from which the result then follows by Proposition 6.14. So suppose $\Phi \subseteq \mathcal{L}$ is finitely satisfiable in $(K, \kappa)$. By compactness, $\Phi$ is satisfiable. Hence there is $(C, \gamma)$ and $c \in C$ such that $c \models_{\gamma} \Phi$. Since $(K, \kappa)$ is final in $\operatorname{Beh}_{\omega}(T)$, there is a mapping $u:(C, \gamma) \rightarrow(K, \kappa) \in$ $\operatorname{Beh}_{\omega}(T)$. By definition of morphisms in $\operatorname{Beh}_{\omega}(T)$, we obtain $u(c) \models_{\kappa} \Phi$. Hence $\Phi$ is satisfiable in $(K, \kappa)$.

'if': Let $(K, \kappa)$ be compact and final in $\operatorname{Beh}_{\omega}(T)$ and suppose $\Phi \subseteq \mathcal{L}$ is finitely satisfiable. Then - by finality and by definition of morphisms in $\operatorname{Beh}_{\omega}(T)-\Phi$ is finitely satisfiable in $(K, \kappa)$, hence satisfiable in $(K, \kappa)$ by compactness and Proposition 6.14.

We now proceed to characterise those endofunctors $T$ for which $\operatorname{Beh}_{\omega}(T)$ has a compact final object. Concerning the logics, we need to impose the following

Condition 7.2. The topologies $\tau_{n}$ are compact and Hausdorff. 
Logically, Condition 7.2 means that $\mathcal{L}$ can separate distinct $\omega$-step behaviours and that every property that is semantically implied by a set of formulas is already implied by a finite subset, see Proposition 6.9.

It will turn out that $\operatorname{Beh}_{\omega}(T)$ has a compact final object iff $T$ weakly preserves the limit of its final sequence up to $\omega$. More precisely, we say that $T$ weakly preserves the limit of the sequence $\left(T^{n} 1\right)_{n \in \omega}$, if the cone $\left(T T^{\omega} 1,\left(T p_{n}^{\omega}\right)_{n \in \omega}\right)$ is weakly limiting. ${ }^{4}$

We now show that the carrier of a compact final object in $\operatorname{Beh}_{\omega}(T)$ is isomorphic to $T^{\omega} 1$. This is the crucial step in our proof.

Lemma 7.3. Assume Condition 7.2. If $(K, \kappa)$ is compact and final in $\operatorname{Beh}_{\omega}(T)$, then $\kappa_{\omega}: K \rightarrow T^{\omega} 1$ is iso.

Proof. It follows from the construction of $(K, \kappa)$ that $\kappa_{\omega}$-called $m$ in Diagram (1) - is mono. To see that $\kappa_{\omega}$ is epi consider $t \in T^{\omega} 1$. The elements of the set $\mathcal{S}=\left\{\kappa_{n}^{-1}\left(\left\{p_{n}^{\omega}(t)\right\}\right) \mid n \in \omega\right\}$ are closed (since in a Hausdorff space one-element sets are closed) and non-empty (follows from Corollary 4.7). It follows from $\kappa_{n}^{-1}\left(\left\{p_{n}^{\omega}(t)\right\}\right) \cap \kappa_{m}^{-1}\left(\left\{p_{m}^{\omega}(t)\right\}\right)=\kappa_{\min (n, m)}^{-1}\left(\left\{p_{\min (n, m)}^{\omega}(t)\right\}\right)$ that $\mathcal{S}$ has the finite intersection property. By compactness, there is $k \in \bigcap \mathcal{S}$. Since $\kappa_{n}(k)=p_{n}^{\omega}(t)$ for all $n \in \omega$, it follows $\kappa(k)=t$.

Observing that $T$ weakly preserves the limit of $\left(T^{n} 1\right)_{n \in \mathbb{N}}$ iff $p_{\omega}^{\omega+1}$ has a one-sided inverse $i, p_{\omega}^{\omega+1} \circ i=i d_{T^{\omega}{ }_{1}}$, we are now able to prove the following theorem.

Theorem 7.4. Let $\mathcal{L}$ be a logic of finite rank satisfying Condition 7.2. The final object of $\operatorname{Beh}_{\omega}(T)$ is compact iff $T$ weakly preserves the limit of $\left(T^{n} 1\right)_{n \in \mathbb{N}}$.

Proof. " $\Rightarrow$ ": Let $(K, \kappa)$ be final and compact in $\operatorname{Beh}_{\omega}(T)$. Due to the lemma above, we can define $i=T \kappa_{\omega} \circ \kappa \circ \kappa_{\omega}^{-1}$. It remains to check that indeed $p_{\omega}^{\omega+1} \circ i=$ $p_{\omega}^{\omega+1} \circ T \kappa_{\omega} \circ \kappa \circ \kappa_{\omega}^{-1}=\kappa_{\omega} \circ \kappa_{\omega}^{-1}=i d_{T^{\omega} 1}$.

" $\Leftarrow "$ ": Let $p_{\omega}^{\omega+1} \circ i=i d_{T^{\omega} 1}$. It was shown in Corollary 4.5 that $\left(T^{\omega} 1, i\right)$ is final in $\operatorname{Beh}_{\omega}(T)$. It is compact since $T^{\omega} 1$ is the limit of compact Hausdorff spaces and the induced topology on a limit of compact Hausdorff spaces is compact Hausdorff (see [5] 3.2.13).

Remark 7.5. An inspection of the proof shows that " $\Rightarrow$ " also holds for logics of rank $\omega$. Moreover, for " $\Rightarrow$ ", we can weaken Condition 7.2 and only require that elements of $T^{n} 1, n<\omega$, are closed wrt. $\tau_{n}$. On the other hand, " $\Leftarrow$ " does not hold for logics of rank $\omega$ as can be seen be the example of $\mathcal{L} \mathcal{T} \mathcal{L}$ (Section 2.2). Indeed, $\left\{\bigcirc^{n} p \mid n<\omega\right\} \cup\{\neg \square p\}$ is finitely satisfiable but not satisfiable.

For the cantor space topology, we have the following:

Corollary 7.6. Let T map finite sets to finite sets. The final object of $\operatorname{Beh}_{\omega}(T)$ is compact in the Cantor space topology iff $T$ weakly preserves the limit of $\left(T^{n} 1\right)_{n \in \mathbb{N}}$.

\footnotetext{
${ }^{4} \mathrm{~A}$ weak limit is defined like a limit but the mediating morphism need not be unique.
} 


\section{Definability for Logics of Rank $\omega$}

In this section we prove a Birkhoff-like characterisation result for classes of coalgebras definable by logics of rank $\omega$. The main idea is again to replace Coalg $(T)$ by $\operatorname{Beh}_{\omega}(T)$ and to reuse well-known techniques ${ }^{5}$. We begin by relating morphisms of $\operatorname{Beh}_{\omega}(T)$ and Coalg $(T)$-morphisms.

Proposition 8.1. For any injective $\operatorname{Beh}_{\omega}(T)$-morphism $m:(C, \gamma) \rightarrow(D, \delta)$ there is $\delta^{\prime}$ such that $m:(C, \gamma) \rightarrow\left(D, \delta^{\prime}\right)$ is a Coalg $(T)$-morphism and $i d_{D}$ : $\left(D, \delta^{\prime}\right) \rightarrow(D, \delta)$ is a $\operatorname{Beh}_{\omega}(T)$-morphism.

Proof. Let $L$ be the image of $m, m_{0}: C \rightarrow L$ the induced mapping, and $R=D \backslash L$. Define $\lambda: L \rightarrow T D$ as $T m \circ \gamma \circ m_{0}^{-1}$ and $\delta^{\prime}: D \cong L+R \rightarrow T D$ as $[\lambda, \delta \circ i n r]$. Then $m$ is a Coalg $(T)$-morphism since $\delta^{\prime} \circ m=\lambda \circ m_{0}=T m \circ \gamma$. To see that $i d_{D}$ is a $\operatorname{Beh}_{\omega}(T)$-morphism assume $\delta_{n}^{\prime}=\delta_{n}$ and consider the following two cases: for $d \in L, \delta_{n+1}^{\prime}(d)=\gamma_{n+1}\left(m^{-1}(d)\right)=\delta_{n+1}\left(m\left(m^{-1}(d)\right)\right)=\delta_{n+1}(d)$; for $d \in R, \delta_{n+1}^{\prime}(d)=T \delta_{n}^{\prime} \circ \delta^{\prime}(d)=T \delta_{n} \circ \delta(d)=\delta_{n+1}(d)$.

In addition to the classical closure operators we need a further one accounting for the restricted expressiveness of logics of rank $\omega$.

Definition 8.2. Let $\mathcal{L}$ be a logic and $(K, \kappa)$ the final object in $\operatorname{Beh}_{\omega}(T)$. Define a relation $\sim \sim_{\omega}^{\mathcal{L}}$ on coalgebras via

$$
(C, \gamma) \sim_{\omega}^{\mathcal{L}}(D, \delta) \Longleftrightarrow \mathrm{cl}\left(!_{C}(C)\right)=\mathrm{cl}\left(!_{D}(D)\right)
$$

where ! denotes the morphisms given by finality of $(K, \kappa)$ and $\mathrm{cl}$ denotes the closure w.r.t. $\left(K, \tau_{K}\right)$ (Definition 6.1).

Theorem 8.3. Let $\mathcal{L}$ be a logic of rank $\omega$. A class $\mathcal{B}$ of $T$-coalgebras is definable by a set of formulae iff $\mathcal{B}$ is closed under coproducts, subcoalgebras, and $\sim \sim_{\omega}^{\mathcal{L}}$.

Proof. Since $\mathcal{L}$ is of rank $\omega$, Proposition 5.3 shows, that $\mathcal{L}$ can be represented by $\llbracket \cdot \rrbracket_{\mathrm{K}}: \mathcal{L} \rightarrow \mathcal{P} K$, where $\mathrm{K}=(K, \kappa)$ denotes the final object of $\operatorname{Beh}_{\omega}(T)$. Recall that $\mathrm{C}=(C, \gamma)=\varphi \Leftrightarrow !_{\mathcal{C}}(C) \subseteq \llbracket \varphi \rrbracket$ where $!_{C}$ is the morphism given by finality. From this observation 'only if' follows easily (for closure under $\sim_{\omega}^{\mathcal{L}}$ recall Proposition 6.10).

For 'if', note first that the assumed closure conditions imply: $C \rightarrow D$ a surjective $\operatorname{Beh}_{\omega}(T)$-morphism only if $\mathrm{C} \in \mathcal{B} \Leftrightarrow \mathrm{D} \in \mathcal{B}$; and $\mathrm{C} \rightarrow \mathrm{D}$ a $\operatorname{Beh}_{\omega}(T)$-morphism only if $\mathrm{D} \in \mathcal{B} \Rightarrow \mathrm{C} \in \mathcal{B}$ (use Proposition 8.1); and for a class $\left\{f_{i}: \mathrm{C}_{i} \rightarrow \mathrm{D} \mid\right.$ $i \in I\}$ of $\operatorname{Beh}_{\omega}(T)$-morphisms with $\mathrm{C}_{i} \in \mathcal{B}$, the union of the images of the $f_{i}$ carries a coalgebra structure and is in $\mathcal{B}$ (use Proposition 3.7). Let $\mathrm{S}=(S, \sigma)$ be the coalgebra given by the union of the images of all $!_{D}: D \rightarrow K, D \in \mathcal{B}$. By Proposition 6.10, cl is expressible in $\mathcal{L}$ by a set of formulae $\Phi$. We show that $\mathcal{B}=\operatorname{Mod}(\Phi)$. For $\mathrm{D} \in \mathcal{B}$ we have, by definition of $S, \mathrm{D} \models \Phi$. To show $\mathcal{B} \supseteq \operatorname{Mod}(\Phi)$, define $\overline{\mathrm{S}}=(\bar{S}, \bar{\sigma})$ as the largest subcoalgebra of $\mathrm{cl}(S)$. Since

\footnotetext{
${ }^{5}$ See e.g. [1], Chapter 16 for a textbook presentation and [14], Chapter 2 for applications to modal logic.
} 
$S \subseteq \bar{S} \subseteq \operatorname{cl}(S)$, it follows $\operatorname{cl}(S)=\operatorname{cl}(\bar{S})$, hence $S \sim_{\omega}^{\mathcal{L}} \overline{\mathrm{S}}$. Since $\mathcal{B}$ is closed under images and coproducts, $\mathcal{B}$ is also closed under unions, hence $\mathrm{S} \in \mathcal{B}$, hence $\overline{\mathrm{S}} \in \mathcal{B}$. Now assume $\mathrm{C} \models \Phi$, that is, $!_{\mathrm{C}}(C) \subseteq \mathrm{cl}(S)$ and hence $!_{\mathrm{C}}(C) \subseteq \bar{S}$, i.e. there is a morphism $C \rightarrow \bar{S}$. Since $\mathcal{B}$ is closed under domains of morphisms, $C \in \mathcal{B}$.

For the Cantor space, we obtain:

Corollary 8.4. Let $\mathcal{L}$ be a finite step expressive logic of finite rank. A class $\mathcal{B}$ of $T$-coalgebras is definable by a set of formulas iff $\mathcal{B}$ is closed under coproducts, subcoalgebras, and $\sim_{<\omega}$.

\section{Conclusions and Related Work}

We have studied definability and compactness for finitary coalgebraic modal logic. The main instrument through which finitary logics have been studied is the terminal sequence and the shift from the category $\operatorname{Coalg}(T)$ to the category $\operatorname{Beh}_{\omega}(T)$.

In this category, points (or states) can be distinguished iff their finite behaviour differs. Also, Beh $\omega(T)$ provides the right framework in which the construction of canonical models can be generalised to a coalgebraic setting. The main handle which allows to formalise the finitary character of the logics considered is to identify finitary predicates with subsets of $T^{n} 1$, where $n$ is a finite ordinal. The idea of interpreting formulae on the elements $T^{n} 1$ of the terminal sequence was already used in [21]. The same idea (without the restriction to finite ordinals) also prevails in Moss [20]. There, formulae are constructed using infinitary conjunctions (which do not change the degree of the formulae) and the application of the signature functor $T$ (increasing the degree of the constructed formulae by 1 ).

The signature functors (and hence the logics) which have been discussed in the present paper are all one-sorted. The passage to multi-sorted signatures, i.e. endofunctors Set ${ }^{n} \rightarrow \mathrm{Set}^{n}$ is standard and allows to include the logics discussed in $[10,26]$, which also rely on (syntactically defined) predicate liftings. Since the endofunctors discussed in loc. cit. are all $\omega$-accessible, final coalgebras and canonical models coincide for these logics (which is also reflected by the fact that they are strong enough to characterise behavioural equivalence).

A coalgebraic representation of the Cantor discontinuum has also been given in [24] in the category of posets. The cantor space topology discussed in the present paper arises in a different way: We start with a final coalgebra on the category of sets, which is then equipped with a natural topology.

\section{Acknowledgements}

We would like to thank the anonymous referees of CMCS'02 for useful comments. Also thanks to Clemens Kupke and Yde Venema for many helpful discussions. 


\section{References}

[1] J. Adámek, H. Herrlich, and G. Strecker. Abstract and Concrete Categories. John Wiley \& Sons, 1990.

[2] J. Adámek and V. Koubek. On the greatest fixed point of a set functor. Theoretical Computer Science, 150:57-75, 1995.

[3] Michael Barr. Terminal coalgebras in well-founded set theory. Theoretical Computer Science, 114(2):299-315, June 1993.

[4] P. Blackburn, M. de Rijke, and Y. Venema. Modal Logic. Cambridge University Press, 2001.

[5] Ryszard Engelking. General Topology. Heldermann Verlag, 1989.

[6] Jelle Gerbrandy. Bisimulations on Planet Kripke. PhD thesis, 1999.

[7] Robert Goldblatt. Logics of Time and Computation, volume 7 of CSLI Lecture Notes. Center for the Study of Language and Information, Stanford University, 1992. Second Edition.

[8] Bart Jacobs. The temporal logic of coalgebras via galois algebras. Technical Report CSI-R9906, Computing Science Institute Nijmegen, 1999.

[9] Bart Jacobs. Many-sorted coalgebraic modal logic: a model-theoretic study. Theoretical Informatics and Applications, 35(1):31-59, 2001.

[10] Bart Jacobs. Many-sorted coalgebraic modal logic: a model-theoretic study. Theoret. Informatics and Applications, 35(1):31-59, 2001.

[11] John Kelley. General Topology. Springer, 1955.

[12] Fred Kröger. Temporal Logic of Programs. Springer, 1987.

[13] A. Kurz and D. Pattinson. Definability, canonical models, compactness for finitary coalgebraic modal logic. In Lawrence Moss, editor, Proceedings of CMCS 2002, volume 65.1 of ENTCS. Elsevier, 2002.

[14] Alexander Kurz. Logics for Coalgebras and Applications to Computer Science. $\mathrm{PhD}$ thesis, Ludwig-Maximilians-Universität München, 2000.

[15] Alexander Kurz. A co-variety-theorem for modal logic. In M. Zakharyaschev, K. Segerberg, M. de Rijke, and H. Wansing, editors, Advances in Modal Logic 2. Center for the Study of Language and Information, Stanford University, 2001.

[16] Alexander Kurz. Modal rules are co-implications. In A. Corradini, M. Lenisa, and U. Montanari, editors, Coalgebraic Methods in Computer Science (CMCS'01), volume 44.1 of ENTCS. Elsevier, 2001. 
[17] Alexander Kurz. Specifying coalgebras with modal logic. Theoretical Computer Science, 260:119-138, 2001.

[18] Z. Manna and A. Pnueli. The Temporal Logic of Reactive and Concurrent Systems. Springer, 1992.

[19] Mike Smyth. Topology. In S. Abramsky, D. M. Gabbay, and T. S. E. Maibaum, editors, Handbook of Logic in Computer Science, volume 1. Oxford University Press, 1993.

[20] Lawrence Moss. Coalgebraic logic. Annals of Pure and Applied Logic, 96:277-317, 1999.

[21] Dirk Pattinson. Semantical principles in the modal logic of coalgebras. In Proceedings 18th International Symposium on Theoretical Aspects of Computer Science (STACS 2001), volume 2010 of LNCS, Berlin, 2001. Springer.

[22] Dirk Pattinson. Coalgebraic Modal Logic: Soundness, Completeness and Decidablility. Technical report, Institut für Informatik, LMU München, 2002.

[23] Dirk Pattinson. Expressive logics for coalgebras via terminal sequence induction. Technical report, Institut für Informatik, LMU München, 2002.

[24] Dusko Pavlovic and Vaughan Pratt. On coalgebra of real numbers. In B. Jacobs and J. Rutten, editors, Electronic Notes in Theoretical Computer Science, volume 19. Elsevier Science Publishers, 2000.

[25] Martin Rößiger. Coalgebras and modal logic. In Horst Reichel, editor, Coalgebraic Methods in Computer Science (CMCS'00), volume 33 of Electronic Notes in Theoretical Computer Science, pages 299-320, 2000.

[26] Martin Rößiger. Coalgebras and Modal Logic. In H. Reichel, editor, Coalgebraic Methods in Computer Science (CMCS'2000), volume 33 of ENTCS, 2000.

[27] Martin Rößiger. From modal logic to terminal coalgebras. Theoretical Computer Science, 260:209-228, 2001.

[28] J.J.M.M. Rutten. Universal coalgebra: A theory of systems. Theoretical Computer Science, 249:3-80, 2000. First appeared as technical report CS R 9652, CWI, Amsterdam, 1996.

[29] Johan van Benthem. Modal Logic and Classical Logic. Bibliopolis, Naples, 1983.

[30] Steven J. Vickers. Topology Via Logic, volume 5. Cambridge University Press, 1988. 
[31] James Worrell. Terminal sequences for accessible endofunctors. In B. Jacobs and J. Rutten, editors, Coalgebraic Methods in Computer Science (CMCS'99), volume 19 of Electronic Notes in Theoretical Computer Science, pages 39-53, 1999.

[32] James Worrell. On Coalgebras and Final Semantics. PhD thesis, Oxford University Computing Laboratory, 2000. 